\title{
Designing incentive packet relaying strategies for wireless ad hoc networks with game theory
}

\author{
Lu Yan and Stephen Hailes \\ University College London, Dept. of Computer Science \\ Gower Street, London WC1E 6BT, UK \\ \{1.yan|s.hailes\}@cs.ucl.ac.uk
}

\begin{abstract}
In wireless ad hoc networks, nodes are both routers and terminals, and they have to cooperate to communicate. Cooperation at the network layer means routing (finding a path for a packet), and forwarding (relaying packets for others). However, because wireless nodes are usually constrained by limited power and computational resources, a selfish node may be unwilling to spend its resources in forwarding packets that are not of its direct interest, even though it expects other nodes to forward its packets to the destination. In this paper, we propose a game-theoretic model to facilitate the study of the non-cooperative behaviors in wireless ad hoc networks and analyze incentive schemes to motivate cooperation among wireless ad hoc network nodes to achieve a mutually beneficial networking result.
\end{abstract}

\section{Introduction}

In a wireless ad hoc network, intermediate nodes on a communication path are expected to forward packets of other nodes so that nodes can communicate beyond their wireless transmission range. Typical examples of the wireless ad hoc networks include military communication or emergency response scenarios, where all participating nodes belong to the same authority, share a common goal, and are therefore motivated to act cooperatively to provide network services [1]. However, since wireless ad hoc networks are increasingly deployed in daily life scenarios such as inter-vehicle communication [2] and Internet access for remote areas [3], the participating nodes most unlikely belong to a single authority and no longer share a common goal any more. Cooperation in such networks cannot be assumed since it has been commonly accepted that network nodes in the above scenarios have the freedom to make decisions in their own best interests.

Since wireless nodes are usually constrained by limited power and computational resources, a selfish node may be unwilling to spend its resources in forwarding packets which are not of its direct interest, even though it expects other nodes to forward its packets to the destination. This is particularly true in typical wireless sensor network nodes [4], where battery-power is a scarce resource and forwarding is an energy-consuming network activity that will shorten a node's lifetime. Even in an ideal case in which nodes have no power and computational constraints, forwarding packets consumes a portion of bandwidth and processing time available to the 
forwarding nodes. Thus, the forwarding cost is not zero; a reasonable move for a node which does not belong to a central authority is to drop the packets belonging to any other node but itself, i.e., to act selfishly. In the worst case, assuming every node is selfish, this behavior will have a collective effect to bring down the communication mechanism of the whole ad hoc network, and result to a tragedy-of-the-commons [5].

In this paper, we propose a game-theoretic model to facilitate the study of the selfish behaviors in wireless ad hoc networks and analyze incentive schemes to motivate cooperation among wireless ad hoc network nodes to achieve a mutually beneficial networking result. The rest of this paper is organized as follows. Section II reviews the literature. In Section III we study two classical strategies under the game theoretic framework. We present a novel incentive strategy for wireless nodes in Section IV and then conclude the paper in Section V.

\section{Related work}

Non-cooperative issues in ad hoc networks have drawn considerable attention over the past few years, and it has been shown that the presence of selfish nodes degrades the overall performance of a non-cooperative ad hoc network [6].

Ideally, a right amount of incentives should be provided to motivate cooperation among wireless ad hoc network nodes to achieve a mutually beneficial networking result. Briefly, participating nodes should be rewarded for cooperative behaviors and be punished for non-cooperative behaviors. The incentive schemes for packet forwarding in the literature basically fall into two categories, namely, trust-based schemes and price-based schemes.

Trust-based schemes use trustworthiness and reputation information in routing to enforce the cooperation among nodes. In [6], participating nodes are designed to perform monitoring to overhear the packet retransmission and avoid transmission to misbehaving nodes. CONFIDANT [7] distributes trustworthy information among those participating nodes, and every node is supposed to keep a reputation list of all its previous interacted nodes. A subjective logic trust model is proposed in [8] for the mapping between the evidence space and the trust space, and further applied to the trusted protocol for wireless ad hoc networks. This design is similar in [9], but with more technical details and data results. More recent approaches such as [10] and [11] evaluate trust level of a node by aggregating feedback information from its neighbors to reduce the communication overhead. STRUDEL [12] is a distributed framework that tackles the problem of free-riders in Coalition Peering Domains by using a Bayesian trust model to dynamically select and isolate malicious peers.

Price-based schemes introduce services charges to the packet transmission process and usually use micro-payment to compensate the resource consumption incurred from the transmission. A virtual currency NUGLET [13] is introduced by L. Buttyan and J.-P. Hubaux as an economic incentive proposal. Each intermediate node buys a packet for some nuglets, and sells it to the next one for more nuglets. Therefore a node increases its nuglet amount during packet forwarding. SPRITE [14] applied the same idea, but with a credit based system and a central clearing banking service, to alleviate the need to carry the real currency. A priced priority forwarding scheme was 
studied in [15], where as pricing mechanism allows the nodes to arbitrarily set the cost of priority forwarding of a packet. APE [16] is a virtual economy scheme to encourage the intermediate nodes to reveal the cost of packet forwarding, and thus to choose a cost-efficiency route. A general discussion about micro-payment schemes is available [17], but not specific to the wireless ad hoc networks.

Since a node's behaviors in wireless ad hoc networks mirror the real world decision making process, game theory models and designs have been applied in the wireless ad hoc networks literature. In [18], V. Srinivasan et al. proposed a game playing strategy to achieve Nash equilibrium which converges to the rational and Pareto optimal. The fairness issue was studied in [19] for bandwidth allocation in ad hoc networks and a game theory model for intrusion detection in wireless ad hoc networks was discussed in [20]. A survey on applying game theory to wireless ad hoc networks is available [21]. Some of the basic analysis of forwarding in single-stage and repeated-game scenarios can be founded on [28, 29]. However, the existing works are either not explicit or too general. In this paper, we propose a formal action model of wireless ad hoc network nodes based on game theory. Since all nodes have to periodically choose an action (e.g. forwarding or dropping), this is a repeated game for all the participants. Because of the complexity of the problem, we restrict our analysis to a static network scenario in this paper.

\section{Game theoretic model}

Let us first consider the simplest forwarding scenario which consists of two nodes $i$ and $j$, where $i$ wants $j$ to forward a packet to other nodes. The denotations used in the following discussions are summarized in Table 1.

Table 1. Denotation list

\begin{tabular}{|l|l|}
\hline$r$ & Reward \\
\hline$s$ & Resource \\
\hline$b$ & Benefit \\
\hline$t$ & Time \\
\hline$d$ & Discount rate \\
\hline$f$ & Forward probability \\
\hline$-i$ & Node's opponent \\
\hline$h$ & Tolerance threshold \\
\hline
\end{tabular}

\subsection{The single-stage game}

We assume that $j$ has freedom to decide whether to forward or drop the packet. We also assume there is a reward mechanism such that for each successful forwarding, $i$ will get its reward of value $r$ for enforcing $j$ to forward packets; and meanwhile, $j$ will consume its resource of value $s$ to complete the forwarding.

Further, we define the interaction model between nodes as bi-directional, i.e., while $j$ tranmits $i$ 's packet, $i$ shall transmit $j$ 's packet simultaneously. Since bi-directional 
wireless communication is never physcially simultaneous, this setting has implication on the physical layer in which the two transmissions in one interaction should be independent, i.e., nodes should make decisions on forwarding or dropping at the same time.

We can see the above setting is a single stage prisoner's dilemma [22], where the motivation for a node to participate in this game playing is to maximize its benefit and the action it can take is to decide whether to forward or drop. For this game, the benefits accrued by each node for every strategy profile are tabulated in Table 2.

Table 2. Benefit vs. strategy

\begin{tabular}{|l|l|l|}
\hline & Forward & Drop \\
\hline Forward & $r-s, r-s$ & $-s, r$ \\
\hline Drop & $r,-s$ & 0,0 \\
\hline
\end{tabular}

In this typical single stage prisoner's dilemma, the best strategy for a participating node is to drop the packet, since this action will maximize its benefit. We can further prove that mutual dropping will lead to Nash equilibrium: since the benefit function of each node is monotonically increasing with dropping actions and the maximized benefit of a node is $r$, dropping is a dominating strategy in this case. In other words, no node can gain more profit by cooperation with others. Putting into the wireless ad hoc networking context, it implies that selfish actions are actually encouraged but this will lead to zero throughput of the whole network.

\subsection{The repeated game}

If the packet forwarding game is played only once, there is no way we can achieve cooperation among these participating nodes; but in a real world setting, all nodes have to periodically choose an action (e.g. forwarding or dropping), and it becomes a repeated game for all the participating nodes. Similarly to the single stage game above, it achieves a non-optimum equilibrium when played repeatedly; however, suboptimum equilibriums are achievable, provided that players do not know a priori how many repetitions of the game there will be.

Since a repeated game is path-dependent, a node has to take consideration of the present action's impact on its future consequence [23]. We extend our previous model to reflect the impact of past event on future actions: assume that time $t$ is discrete and divided in frames like $t_{1}, t_{2} \ldots t_{n}$. A node $i$ will make a decision at each stage $t_{n}$ of the game, but its benefit gained at each stage will be discounted by a rate at $d$ to reflect a past action's impact factor over time [24].

A participating node in this game takes actions by adjusting its attitude towards forwarding. Explicitly, we denote a node's probability to forward a packet as $f$, where in this case $f=0$ means dropping all the requested packets and $f=1$ mean forwarding all the requested packets. Let $i$ denote the playing node's id and $-i$ denote its opponent, the benefit of a node for playing this game at a time frame $t_{n}$ is $b_{i}^{t_{n}}=f_{-i}^{t_{n}} r-f_{i}^{t_{n}} s$. The final benefit of a node finishing the game is 
$B_{i}=\sum_{n=0}^{\infty} d^{n}\left(f_{-i}^{t_{n}} r-f_{i}^{t_{n}} s\right)$, where $d$ is the discount rate. We study two classical strategies [23] and their sub-optimum equilibrium conditions.

\subsubsection{Tit For Tat}

TFT (Tit For Tat) is a highly effective strategy in game theory for the iterated prisoner's dilemma [23]. A player using this strategy will initially cooperate, and then respond in kind to an opponent's previous action. If the opponent previously was cooperative, the player is cooperative. If not, the player is not. We model the TFT strategy as follows:

$$
f_{i}^{t_{n}}=\left\{\begin{array}{c}
1, n=0 \\
f_{-i}^{t_{n-1}}, n>0
\end{array}\right.
$$

TFT provides incentives to cooperate since one player's present move will have impact on its future consequence. Let's study the node dynamics in this strategy.

Assume all participating nodes are adopting TFT at the bootstrapping stage. It is obvious that this cooperation will continue as the forwarding probability will remain 1 and thus all packets will be forwarded. If at some point a node $i$ unilaterally changes its forwarding probability to $f_{i}^{t_{0}}=p$ (e.g. due to a physical transmission failure), its opponent will copy its behavior and set its forwarding probability to $f_{-i}^{t_{1}}=p$; but the node $i$ itself follows its opponent's previous behavior, thus its own forwarding probability will be set back to $f_{i}^{t_{1}}=1$. The above process will continue repeatedly and in the end we will see an alternate changing sequence as follows:

$$
\begin{aligned}
f_{i}^{t_{n}} & =\{p, 1, p, 1, \cdots\} \\
f_{-i}^{t_{n}} & =\{1, p, 1, p, \cdots\}
\end{aligned}
$$

We can calculate the final benefit of node $i$ for playing this game:

$$
\begin{aligned}
B_{i} & =\sum_{n=0}^{\infty} d^{n}\left(f_{-i}^{t_{n}} r-f_{i}^{t_{n}} s\right) \\
& =[(r-p s)+d(p r-s)] *\left(1+d^{2}+d^{4}+\cdots\right) \\
& =\frac{(r-p s)+d(p r-s)}{1-d^{2}}
\end{aligned}
$$

A node will adopt this strategy if and only if it will gain profit from it, i.e. $B_{i} \geq 0$. We solve Equation 3 and derive the boundary condition as: 


$$
\frac{r}{s} \geq \frac{d+p}{1+d p}
$$

\subsubsection{Grim Trigger}

Grim Trigger (GT) is a trigger strategy in game theory for a repeated game [23]. Initially, a player using GT will cooperate, but as soon as the opponent defects (thus satisfying the trigger condition), the player using GT will defect for the remainder of the iterated game.

Since a single defect by the opponent triggers defection forever, GT is the most strictly unforgiving of strategies in an iterated game. The implication in the network design is that once a node is misbehaved, it will be isolated permanently. We model GT as follows:

$$
f_{i}^{t_{n}}=\left\{\begin{array}{c}
1, n=0 \\
1, \forall m<n: f_{-i}^{t_{m}} \geq h \text { and } n>0 \\
0, \text { all else }
\end{array}\right.
$$

where $h$ is introduced as the tolerance threshold and the trigger condition is $f<h$.

We will then discuss the sub-optimum equilibrium condition for GT. Suppose all nodes are adopting GT at the bootstrapping stage, and the cooperation continues until some point when a node $i$ unilaterally changes its forwarding probability to $f_{i}^{t_{0}}=p$. Depending on the degree of deviation, its opponent will respond with GT: (1) a slight degree of deviation, i.e. $p \geq h$, will be tolerated; and it will still forwards all packets (2) a bigger deviation, i.e. $p<h$ will trigger the punishment, and it will from now on drop all the packets forever. If we consider the worst case $p<h$, the forwarding probability sequence will be

$$
\begin{aligned}
f_{i}^{t_{n}} & =\{p, 1,0,0, \cdots\} \\
f_{-i}^{t_{n}} & =\{1,0,0,0, \cdots\}
\end{aligned}
$$

Let us calculate the final benefit of node $i$ for playing this game:

$$
\begin{aligned}
B_{i} & =\sum_{n=0}^{\infty} d^{n}\left(f_{-i}^{t_{n}} r-f_{i}^{t_{n}} s\right) \\
& =r-p s-d s
\end{aligned}
$$

Using the condition $B_{i} \geq 0$, we get the boundary condition for a node to apply this strategy: 


$$
\frac{r}{S} \geq d+p
$$

\section{Proposed incentive strategy}

Any breach of cooperation in TFT or GT results in either all packets being dropped or some fraction of packets being dropped. In other words, fully cooperative is never the Nash equilibrium point with TFT or GT. Meanwhile, the boundary conditions from Section III imply that cooperation can be achieved in a network of selfish nodes, given network parameters (e.g., earning-cost ratio) carefully designed. Besides, in a real world communication process, network fluctuations and measurement errors may trigger unjust punishments, and those punishments may have irrevocable future effects in some strategies; in designing a practical strategy for real world networking, recovery mechanism shall be taken into serious considerations.

From the above analysis, we can see that a strategy without recovery mechanism is not stable in practice. We aim to design a new strategy that reciprocates both cooperation and defection, but shall still be able to rebuild cooperation after a node's unintentional misbehaviors.

It is our hypothesis that an optimal strategy should bear at least the following merits:

--It is good (it starts by cooperating)

--It is retaliating (it returns the opponent's defection)

--It is generous (it forgets the past if the defecting opponent cooperates again)

--It is not memoryless (it utilizes the history information).

Our goal is to propose a strategy that is more adaptive to fluctuations and able to re-achieve full cooperation after a node's misbehaviors. For the case of wireless ad hoc networks we design the Gradual strategy based on the idea of adaptiveness [25]: a player uses cooperation on the initial move and then continues to do so as long as its opponent cooperates. Then after the first defection of the opponent, it defects one time and cooperates two times; after the second defection of the opponent, it defects two times and cooperates two times, ... after the $\mathrm{N}^{\text {th }}$ defection it reacts with $\mathrm{N}$ consecutive defections and then calms down its opponent with two cooperations:

$$
\begin{aligned}
& f_{i}^{t_{0}}=1 ; \mathrm{N}=0 ; \mathrm{n}=1 ; / / \text { start with forward } \\
& \text { while }(\mathrm{n}++)\{/ / \text { time-series } \\
& \qquad \begin{aligned}
\text { if }\left(f_{-i}^{t_{n-1}}<h\right)\{/ / \text { opponent drop } \\
\mathrm{N}++; \mathrm{M}=\mathrm{n}+\mathrm{N} ; \\
\text { while }(\mathrm{n}<\mathrm{M}+1) / / \text { respond with } \mathrm{N} \text { drops }
\end{aligned}
\end{aligned}
$$




$$
\begin{gathered}
\left\{f_{i}^{t_{n}}=0 ; \mathrm{n}++;\right\} \\
f_{i}^{t_{n}}=1 ; \mathrm{n}++; f_{i}^{t_{n}}=1 ; / / \text { two forwards } \\
\} \\
\text { else } f_{i}^{t_{n}}=1 ; / / \text { otherwise forward }
\end{gathered}
$$

\}

Using the same assumptions and settings in analyzing the previous strategies, we can derive a forwarding sequence after a node's misbehavior as follows:

$$
\begin{aligned}
f_{i}^{t_{n}} & =\{p, 1,0,1,1, \cdots\} \\
f_{-i}^{t_{n}} & =\{1,0,1,1,1, \cdots\}
\end{aligned}
$$

We shall admit this is the simplest case with Gradual, and Equation 9 may become very complex when a node/opponent misbehaves again. Though we can still use the previous mathematics analysis method to find out the sub-optimum equilibrium for Gradual, the boundary condition itself shall take a complex form whose implication is no longer intuitive. Thus it is our hypothesis that simulation is a better way to understand the dynamics in this strategy.

In a real world networking environment, nodes may take divergent strategies. For instance, (1) a group of nodes may employ TFT, and the other may use GT; (2) a node may take TFT in the bootstrapping stage, but it may switch to GT in a later stage. In other words, we need to study the mixed-strategy situations. However, such situations are complex in nature and simulation may be a better way to understand the cooperation behavior over time, which is generally believed to be evolutionary [26].

Complex strategies in the iterated prisoner's dilemma have been studied by Axelrod [26] and a computer simulated tournament was proposed to evaluate different strategies when they compete against each other [27]. Typically, game theorists use a round robin tournament to study the behavior of strategies. Each strategy meets all other strategies in this tournament, and its score (i.e., benefit) is recorded for each confrontation. Its final score is the un-discounted sum of all scores, and the strategy's goodness is measured by its final score completing the tournament.

However, the above simulation design only provides the final result and does not reveal any dynamic aspects of strategy behaviors throughout the simulation process. We adopt the idea of ecological evolution and revise the round robin tournament as follows: we model the mixed strategy scenario as an ecological evolution process; at the beginning there is a fixed population of the same quantity of each strategy; a round robin tournament is made and then the population of bad strategies is decreased while good strategies obtain new elements. The simulation is repeated until the population has been stabilized. In this simulation scenario, a good strategy is then the strategy which stays alive in the population for the longest possible time, and in the biggest possible proportion [30]. 
Suppose that, initially, the total population is composed of $M$ strategies $S_{i}$ where $i=1, \ldots, M$. At the generation $n$, each strategy is represented by a certain number of players as $W^{n}\left(S_{i}\right)$; Let $V\left(S_{i} \mid S_{j}\right)$ denote the score (i.e. benefit) when strategy $S_{i}$ meets strategy $S_{j}$, which can be derived, for instance, from Table 2 . The computation of the score $g^{n}\left(S_{i}\right)$ of a player using a selected strategy $S_{i}$ at the generation $n$ is:

$$
g^{n}\left(S_{i}\right)=\sum_{j=1}^{M}\left(W^{n}\left(S_{j}\right) V\left(S_{i} \mid S_{j}\right)\right)-V\left(S_{i} \mid S_{i}\right)
$$

The size of each sub-population $W^{n+1}\left(S_{i}\right)$ at the generation $n+1$ is:

$$
W^{n+1}\left(S_{i}\right)=\left\lfloor\frac{W^{n}\left(S_{i}\right) g^{n}\left(S_{i}\right) \sum_{j=1}^{M} W^{n}\left(S_{j}\right)}{\sum_{j=1}^{M}\left(W^{n}\left(S_{j}\right) g^{n}\left(S_{j}\right)\right)}\right\rfloor
$$

where all divisions shall be rounded to the nearest lower integer, due to the physical meaning of $W^{n}\left(S_{i}\right)$.

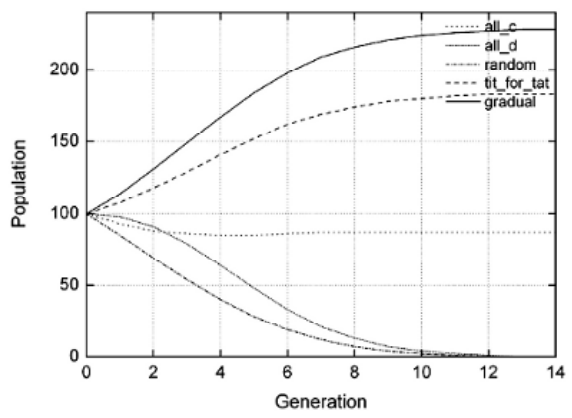

Fig. 1. Simulation result

The simulation consists of 500 random nodes with an even distribution of 5 strategies: all_c (always cooperate), all_d (always defect), random, tit_for_tat, gradual, i.e., initially each strategy is represented by 100 players. $\bar{W}$ e run the simulation algorithm described in section 4.4 .1 , and plot the population changes over time in Fig. 1. It is possible to observe from Figure 1, after 12 generations the all_d and random strategies disappear; gradual has the biggest portion of population, followed by tit_for_tat. This implies that Gradual 
strategy outperforms TFT and proves to be the winning strategy in a mixed strategy environment.

We have then repeated the simulation with more strategy sets and all results show that Gradual is relatively strong, compared to other strategies; and the most significant point is that Gradual has never, or not often, bad scores. Therefore it is our conjecture that Gradual is a good strategy for wireless nodes.

The reason why Gradual outperforms others can be explained as follows: different from all previous memory-less strategies, Gradual keeps a long-term memory about nodes' behaviors and adaptively punishes misbehaviors with history information. Meanwhile it is positive towards rebuilding cooperation with two consecutive cooperations after the punishment.

Actually we can find a number of real life metaphors of this strategy in our daily life, e.g. credit agency vs. debtors, government vs. taxpayers. A node's standing in this strategy can therefore be interpreted in two ways:

- Offensive: a node wants to force its opponent to cooperate, and thus clearly shows that it will be more and more aggressive so that the best choice for its opponent is to cooperate.

- Defensive: a node does not want to be exploited, and thus gets less and less for cooperation. However as it needs services from others, it retries sometimes to reinstall cooperation.

\section{Concluding remarks}

In this paper, we have proposed a game theoretic model to investigate the conditions for cooperation in wireless ad hoc networks. Because of the complexity of the problem, we have restricted ourselves to a static network scenario. The main finding is that a cooperative state in a network of selfish nodes without a central authority is theoretically achievable, given network parameters (e.g. cost-earning ratio, discount rate, tolerance threshold, etc) carefully designed [31].

We have then studied different node strategies within this model, and derived boundary conditions of cooperation for those strategies. As one of the results, we have proposed a refined strategy which is adaptive to fluctuations and able to achieve a full cooperation after a node's unintentional misbehaviors. Due to the memory effect of this strategy, we conducted a simulation study instead of mathematical analysis. Another significant finding in this paper lies in the evaluation of mixed strategy scenarios, where different nodes adopt different cooperation strategies [32].

Throughout the paper we have taken the assumption that packet transmission is unicast. However, recently there has been a plethora of work on using multicast routing protocols in wireless ad hoc networks [33]; we also intend to investigate the packet dynamics in a multicast network and extend the model for it.

\section{Acknowledgment}

This work is partly funded by EPSRC under grant EP/D07696X/1. 


\section{Reference}

1. L. Buttyan and J.-P. Hubaux, "Stimulating Cooperation in Self-organizing Mobile Ad Hoc Networks," in ACM/Kluwer Mobile Networks and Applications, vol. 8, no. 5, pp. 579-592, Oct. 2003.

2. T. Nadeem, S. Dashtinezhad, C. Liao, L. Iftode, "TrafficView: traffic data dissemination using car-to-car communication", in ACM SIGMOBILE Mob. Comput. Commun. Rev., Vol. 8, No. 3, pp. 6-19, July 2004.

3. C.E Perkins, E. M. Belding-Royer, and Y. Sun, "Internet connectivity for ad hoc mobile networks", in International Journal of Wireless Information Networks, April 2002.

4. S. Kumar, A. Arora, T.H. Lai, "On the lifetime analysis of always-on wireless sensor network applications", in Proc. IEEE International Conference on Mobile Ad hoc and Sensor Systems, 2005.

5. G. Hardin, "The Tragedy of the Commons," Science, Vol. 162, No. 3859, pp. 1243-1248, December 1968.

6. S. Marti, T. J. Giuli, K. Lai, and M. Baker, "Mitigating Routing Misbehavior in Mobile Ad Hoc Networks", in Proc. ACM/IEEE International Conference on Mobile Computing and Networking (Mobicom), Boston, August 2000.

7. S. Buchegger and J.-Y. Le Boudec, "Performance analysis of the CONFIDANT protocol," in Proc. International Symposium on Mobile Ad Hoc Networking \& Computing (MOBIHOC 2002), Lausanne, Switzerland, June 2002.

8. X. Li, M.R. Lyu, J. Liu, "A trust model based routing protocol for secure ad hoc networks", in Proc. IEEE Aerospace Conference, 2004.

9. T. Ghosh, N. Pissinou, K. Makki, "Towards designing a trusted routing solution in mobile ad hoc networks", in Mobile Networks and Applications, Volume 10, Issue 6, December 2005.

10. Q. He, D. Wu and P. Khosla, "SORI: A Secure and Objective Reputation-based Incentive Scheme for Ad hoc Networks," in Proc. of IEEE Wireless Communications and Networking Conference (WCNC2004), Atlanta, GA, USA, March 2004.

11. M. T. Refaei, V. Srivastava, L. DaSilva, and M. Eltoweissy, "A Reputation-based Mechanism for Isolating Selfish Nodes in Ad Hoc Networks," in Proc. IEEE Second Annual International Conference on Mobile and Ubiquitous Systems: Networking and Services (MOBIQUITOUS 2005), San Diego, CA, July 2005.

12. D. Quercia, M. Lad, S. Hailes, L. Capra and S. Bhatti, "STRUDEL: Supporting Trust in the Dynamic Establishment of peering coalitions", in Proc. of ACM Symposium on Applied Computing SAC 2006, Dijon, France, April 2006.

13. L. Buttyan and J.-P. Hubaux, "Enforcing service availability in mobile ad-hoc wans", in Proc. IEEE/ACM Workshop on Mobile Ad Hoc Networking and Computing (MobiHOC), Boston, MA, 2000.

14. S. Zhong, J. Chen, and Y. R. Yang, "Sprite: A Simple, Cheat-Proof, Credit-Based System for Mobile Ad Hoc Networks," in Proc. of IEEE Infocom 2003, San Francisco, CA, USA, April 2003.

15. B. Raghavan and A. C. Snoeren, "Priority forwarding in ad hoc networks with selfinterested parties", in Proc. Workshop on Economics of Peer-to-Peer Systems, Berkeley, CA, 2003.

16. L. Anderegg and S. Eidenbenz, "Ad hoc-VCG:A Truthful and Cost-Efficient Routing Protocol for Mobile Ad hoc Networks With Selfish Agents", in Proc. ACM/IEEE International Conference on Mobile Computing and Networking (Mobicom'03), San Diego, CA, 2003.

17. M. Peirce, "Multi-party Micropayments for Mobile Communications", PhD Thesis, Trinity College Dublin, Ireland, Oct. 2000.

18. V. Srinivasan, P. Nuggehalli, C. F. Chiasserini, and R. R. Rao, "Cooperation in wireless ad hoc networks," in Proc. IEEE INFOCOM 2003, San Francisco, CA, Mar./Apr. 2003. 
19. Z. Fang and B. Bensaou, "Fair bandwidth sharing algorithms based on game theory frameworks for wireless ad-hoc networks", in Proc. IEEE INFOCOM 2004.

20. A. Patcha and J.-M. Park, "A Game Theoretic Formulation for Intrusion Detection in Mobile Ad Hoc Networks", in International Journal of Network Security, Vol.2, No.2, Mar. 2006.

21. V. Srivastava, J. Neel, A. B. MacKenzie, R. Menon, L. A. DaSilva, J. E. Hicks, J. H. Reed, and R. P. Gilles, "Using game theory to analyze wireless ad hoc networks," in IEEE Communications Surveys and Tutorials, vol. 7, pp. 46-56, 2005.

22. P. K. Dutta, "Strategies and Games: Theory and Practice," MIT Press, 1999.

23. G. J. Mailath and L. Samuelson, "Repeated Games and Reputations: Long-Run Relationships", Oxford University Press, 2006.

24. M. S. Morgan, D. F. HendryNet, "The Foundations of Econometric Analysis", Cambridge University Press, 1997.

25. B. Beaufils, J.-P. Delahaye and P. Mathieu, “Our Meeting With Gradual: A Good Strategy For The Iterated Prisoner's Dilemma”, in Proc. the Fifth Int'l Workshop on the Synthesis and Simulation of Living Systems, MIT Press, 1997.

26. R. Axelrod and W. D. Hamilton, "The evolution of cooperation", Science, 211: 1390-1396, 1981.

27. R. Axelrod, "The evolution of cooperation", New York, 1984.

28. L. A. DaSilva and V. Srivastava, "Node Participation in Ad-hoc and Peer-to-peer Networks: A Game-theoretic Formulation", Workshop on Games and Emergent Behavior in Distributed Computing Environments, September 18, 2004, Birmingham, U.K.

29. F. Milan, J. J. Jarmillo, and R. Srikant, "Achieving Cooperation in Multihop Wireless Networks of Selfish Nodes", Workshop on Game Theory for Networks, October 14, 2006, Pisa, Italy.

30. M.A. Nowak, A. Sasaki, C. Taylor, D. Fudenberg, "Emergence of cooperation and evolutionary stability in finite populations", Nature, 428: 646-650, 2004.

31. L. Yan, S. Hailes, L. Capra, "Analysis of packet relaying models and incentive strategies in wireless ad hoc networks with game theory", in Proc. IEEE 22nd International Conference on Advanced Information Networking and Applications (AINA'08), Okinawa, Japan, 2008.

32. L. Yan, S. Hailes, "Cooperative Packet Relaying Model for Wireless Ad hoc Networks", in Proc. ACM International Workshop on Foundations of Wireless Ad Hoc and Sensor Networking and Computing (FOWANC'08), co-located with ACM MobiHoc'08, Hong Kong, China, 2008.

33. Z. Li, "Min-Cost Multicast of Selfish Information Flows", in Proc. of the 26th Annual IEEE Conference on Computer Communications (INFOCOM'07), Anchorage, Alaska, May 6-12, 2007. 